\title{
Development and utilisation of the Australian Spinal Cord Injury Register
}

\author{
PJ O'Connor*,1 \\ ${ }^{1}$ Research Centre for Injury Studies, Flinders University, Bedford Park, Adelaide, SA 5042, Australia
}

\begin{abstract}
Objective: To document the development and utilisation of the Australian Spinal Cord Injury Register.

Methods: Review of published material and consultative processes.

Results: The development of a national population based register of spinal cord injury (SCI) was first mooted in Australia in 1987. ${ }^{1}$ Wigglesworth, ${ }^{2}$ in the journal Paraplegia, outlined the planning process for such a register. Later, in the journal Spinal Cord, Blumer and Quine ${ }^{3}$ reported on the identification of the information needs that could be met by a register. However, it was not until 1 July 1995 that the Australian Spinal Cord Injury Register was established, providing the means of achieving the objectives outlined in the Menzies Foundation reports. ${ }^{1,4}$ From that date the registration of cases became an integral part of the routine admission process of the six specialist centres for the treatment of SCI. This, in combination with a process of case note review to identify earlier cases, has ensured that all cases of SCI due to trauma admitted to spinal units have been registered from 1986, providing a significant national database for epidemiological and research purposes.

Conclusions: The main lessons to be learned from the Australian experience are: (1) that it is possible, in cooperation with the Directors of specialist treatment units, to design, implement and maintain a national population-based register, at relatively small financial cost; and (2) that a register provides data for important information and research purposes. As it is unlikely that Australia has unique conditions for the development of a registry, this experience should encourage others. There are however some lessons to be learned, and procedures to be put in place, described in this article, to ensure that data from a registry is useful for advancing the prevention and treatment of SCI.
\end{abstract}

Sponsorship: The register was developed with the financial support of the Australian Institute of Health and Welfare and the Commonwealth Department of Health and Aged Care.

Spinal Cord (2000) 38, 597-603

Keywords: epidemiology; registries; spinal cord injuries; public health; research

\section{Introduction}

Spinal cord injury (SCI) is a significant health condition. Although it is relatively rare, it is important due to the severity of the outcomes in individual, social and economic terms. The prevalent population has been estimated to number in excess of 6000 in Australia. The ongoing cost associated with the longterm care of the newly incident cases has been estimated to be about 200 million Australian dollars per year, based on compensible cases. ${ }^{5}$ As only half the cases are eligible for compensation, the total community cost could well be as high as 400 million Australian dollars per year.

Given that there is still no cure for SCI, prevention should be emphasised. The development of appro-

*Correspondence: PJ O'Connor, Research Centre for Injury Studies, Flinders University, Mark Oliphant Building, Laffer Drive, Bedford Park, Adelaide, South Australia 5042, Australia priate interventions requires information on causal factors as well as on the characteristics of the SCI population. As the outcomes of injury may be the result not only of personal characteristics and behaviours, but also of social and environmental factors, ${ }^{6}$ the collection of detailed information on these factors is required.

The planning of community services for people with SCI requires information on the size of the prevalent population and their health and welfare needs. Survival from SCI may now be approaching that of the non-SCI population, ${ }^{7,8}$ suggesting the potential for an increase in the prevalence of this condition in the Australian community given the existing evidence that the incidence of SCI has not declined. 9 Furthermore, the prevalent population may present hitherto unknown challenges for health and welfare services as the family support and individual physical 
conditioning required for the maintenance of wellbeing succumb to the effects of ageing. Information is required on a range of factors to characterise the health and welfare of the prevalent population, including medical status, quality of life and psychological coping, which are important in themselves, but also reflect on the community support needed for the maintenance of well-being.

The information required for the prevention and control of SCI and the monitoring of the health and welfare of the prevalent population indicates the need for surveillance. Surveillance could be based on routine data sets, such as mortality data and hospital separations data, population survey or a special disease register.

In Australia, routine mortality data has, until recently, been inadequate for the surveillance of SCI. It was not until 1997 that the Australian Bureau of Statistics (ABS) commenced the coding of disease diagnoses, such as SCI, from death certificates. This data has only recently become available, and it is too early to determine whether it will be useful for surveillance.

The nationally available routine hospital separations data is inadequate for the surveillance of SCI. A person who is treated for a suspected SCI because of the presence of vertebral injury but who suffers no neurological loss may be coded as having had an SCI. Also, there is no adequate means of distinguishing the first admission of a newly incident case of SCI from an inter-hospital transfer or a readmission due to complications. Estimates of the incidence of SCI based on the nationally available hospital separations data would therefore be substantially inflated. Furthermore, the scope of the information available is quite restricted and suffers from poor timeliness. Detailed information on the circumstances of injury (eg, causation, time and date) and outcomes (eg, category of impairment) is not available.

Given the low incidence and prevalence of SCI, a population survey to monitor these parameters would need to be very large and regularly repeated, which would be very expensive. If the survey were not repeated regularly, the information provided would quickly become dated and would be limited in the identification of newly emerging conditions. A survey of members of the advocacy or disability support groups for SCI would be inadequate because not all of the people with SCI would be members and there could be problems with the representativeness of any findings based on such a sample.

In Australia, it is possible to identify each hospitalised person who has suffered an SCI from trauma because there are a small number of specialist treatment units nationally (Table 1) and referral of cases with SCI to one of these units is a well-established hospital protocol. Full coverage of the prevalent population, and an interest, by the units, in implementing a common data collection, provided necessary but not sufficient conditions for a
Table 1 Spinal units in Australia

\begin{tabular}{ll}
\hline City & \multicolumn{1}{c}{ Unit } \\
\hline Perth & Sir George Bedbrook Spinal Unit, \\
& Royal Perth (Rehabilitation) \\
& Hospital \\
Adelaide & Spinal Injuries Rehabilitation Unit, \\
& Royal Adelaide Hospital \\
Melbourne & Spinal Unit, \\
& Austin Hospital \\
Sydney & Spinal Unit, \\
& Royal North Short Hospital \& \\
& Royal Rehabilitaion Centre at \\
& Moorong \\
& Spinal Unit, \\
& Prince Henry Hospital \& Prince of \\
& Wales Hospital \\
& Spinal Injuries Unit, \\
Royal Alexandria Hospital
\end{tabular}

national register of SCI. The development process that led to the implementation of the Australian Spinal Cord Injury Register is documented so that other countries can learn from the Australian experience.

\section{History}

The development of a national population based register of SCI was first mooted in Australia in 1987. ${ }^{1}$ Wigglesworth, ${ }^{2}$ in the journal Paraplegia, outlined the planning process for such a register. Two symposia were held, in 1987 and 1988, under the auspices of the Menzies Foundation, a non-political organisation that was formed to promote the health and fitness of the Australian community. The first of these, Towards Prevention of Spinal Injury, ${ }^{1}$ reviewed what was then known about the incidence and cost of spinal injury in Australia. The first recommendation to come from this meeting was that a registry of spinal injury should be established in Australia to identify areas where preventive measures might be successful and also to monitor the effects of such measures. It also recommended a second meeting of appropriate experts to develop the statistical and other details. At the second meeting, Towards a Registry of Spinal Injury, ${ }^{4}$ the aims of the proposed registry were defined. Amongst these were that a register of spinal injury would provide routine information on the incidence, causation, morbidity and mortality, outcomes and long term consequences of, and needs of people with SCI. It was recognised that additional and more detailed data collections based on the register of cases would need to be established for research purposes. One of the recommendations of the report of the second meeting was that a committee to plan and develop the registry should be established, and that it would define the core data set for the proposed register. In order to enhance 
the quality of the data set, declaration of SCI as a notifiable disease was considered. Unfortunately, the proposed committee did not meet, the core data set was not defined, and SCI was not made a notifiable disease.

Although the planning process for a registry was not completed, the symposia had the benefit of drawing attention to the importance of SCI at the highest levels of the health ministry. This encouraged the expansion of a State data collection, based in New South Wales and developed by an Actuary, Mr John Walsh, into a national data collection from 1986. The collection had as its principal objective the evaluation of care received by people following their return to the community. It was not developed on the basis of a national assessment of information needs and did not fulfil all of the objectives envisaged in the Menzies Foundation reports. At the end of 1991, the Motor Accidents Authority of New South Wales (the major supporter) and the Disability Services Program of the (then) Commonwealth Department of Community Services and Health, declined to renew their funding of the project. Subsequently data collection at national level ceased.

Recognising the importance of implementing a registry in the way envisaged in the Menzies Foundation reports, particularly that a data collection must service clear information needs, the Australian Institute of Health and Welfare (AIHW) National Injury Surveillance Unit (NISU) funded a review of the needs and opportunities for SCI surveillance. This review was finalised in 1995 and Blumer and Quine ${ }^{3}$ have published the results in the journal Spinal Cord. In brief, the review identified five broad areas of information demand: (i) service evaluation and planning, (ii) epidemiology, (iii) prevention, (iv) external demands, and (v) research.

The review recommended that a register of incident cases be established and managed by the NISU, then a unit of the AIHW and now a program of the Flinders University Research Centre for Injury Studies.

\section{Methods}

The Menzies Foundation reports ${ }^{1,4}$ and the reviews by Blumer $^{3,10}$ provided an overview of the possible functions of an SCI register, provided guidance as to the types of information that might be collected and specified the types of uses for the data that could be compiled. However, the practicalities involved with its implementation were not addressed. These were developed and negotiated by the author of the present article and his project officer, Dr Raymond Cripps, and the Directors of the spinal units, working in collaboration. The key factors in the success of the collaboration were:

(1) The enthusiasm of the information scientists and spinal unit Directors that a register be established within a short time frame.
(2) Recognition that the minimum data set (MDS) would necessarily be a compromise of the full information requirements of the units.

(3) Inclusion of data items in the MDS only where the intended use could be clearly specified and justified.

(4) Piloting in a single unit, with feedback to other units, so that the development issues could be ironed out efficiently.

(5) Regular feedback to the Directors and their staff on progress and outcomes, especially the prompt release and distribution of a national annual statistical report based on the register.

\section{Results}

Over a period of 6 months, the details of the register were decided. An outline of the processes and outcomes is presented below.

\section{Objective}

The agreed purpose of the register was identified as the collection of information on the incidence, causation, morbidity and mortality, outcomes and long term consequences of, and needs of people with, SCI, as stated in the Menzies Foundation symposium report. ${ }^{1}$

\section{Case definition}

In order to facilitate national and international comparisons, the case definition that was adopted for registration of traumatic cases of SCI was the CDC clinical definition:

' ... a case of spinal cord injury is defined as the occurrence of an acute, traumatic lesion of neural elements in the spinal canal (spinal cord and cauda equina), resulting in temporary or permanent sensory deficit, motor deficit, or bladder/bowel dysfunction'. ${ }^{11}$

\section{The minimum data set}

The core set of data items used for registration purposes was chosen as a minimal set of items well suited to a paper-reporting system. For data comparability, they were chosen from national and international data standards ${ }^{12-14}$ issued by authorities such as the American Spinal Injury Association, World Health Organisation and the AIHW.

The agreed data set included the following types of information: hospital and patient identifiers, social and demographic items, service and administrative items, and basic clinical and public health information. The neurological level of injury and degree of impairment was coded according to the American Spinal Injury Association (ASIA) International Standards for Neurological and Functional Classification of Spinal Cord Injury (Revised 1996). ${ }^{12}$ The level of independence in undertaking daily life activities was measured according to the Functional Independence Measure 
(FIM). ${ }^{15}$ The external cause and injury diagnosis data was coded according to the International Classification of Diseases (ICD-9-CM, Australian revision). ${ }^{13}$ The other items in the agreed data set conformed to the AIHW National Health Data Dictionary, which incorporates the NISU National Data Standards for Injury Surveillance. ${ }^{14}$

Spinal unit Directors agreed to incorporate the collection of these items into the routine admission process at each unit and forward their data to NISU.

\section{Information and research services}

NISU agreed to provide regular case lists and statistical summaries to each spinal unit; a widely distributed annual statistical report on the incidence of SCI; as well as an ad hoc information service. Furthermore, it was envisaged that once the register was established it would provide the basis for more detailed data collection and research focusing, for example, on long-term outcomes and survival. Individual units were encouraged to collect information beyond the minimum data set for their own internal purposes.

\section{Ethics committee approval}

Ethics committee approval was required from each spinal unit hospital for patient identified data to be made available to NISU. Patient consent was required for newly incident cases. However, for prevalent cases of SCI that were not already registered, the ethics committees did not require patient consent. This decision was made on the basis of the following factors: (i) the impracticality of attainment of consent many years after SCI, (ii) the importance of complete national data on SCI, (iii) the high consent rate obtained by the national data collection that operated during the period 1986 to 1991, and (iv) the strict constraints imposed on NISU to protect patient confidentiality and control the use of register.

The confidentiality and privacy of patient information supplied to NISU was assured by its adherence to the AIHW's standards and procedures on legal, ethical and professional matters. The AIHW is governed by an Act of Parliament (the 'Australian Institute of Health and Welfare Act 1987') which specifies how privacy and confidentiality must be protected. ${ }^{16}$ It is also subject to requirements of the Federal 'Privacy Act 1988'. The AIHW Ethics Committee approved plans for the utilisation of the data.

In order to protect the confidentiality of patient data, all data forms received were stored in secure lockable filing cabinets with access being restricted to two authorised NISU staff. The guiding principles with respect to provision of information regarding SCI, which formed part of the patient and spinal unit agreement over the supply of data to NISU, were: (i) that patient identified data only be made available to the source spinal unit and (ii) that aggregated data tables distributed more widely needed to be reported in such a way that individuals could not be identified.

\section{Piloting}

Following receipt of Ethics Committee approvals, NISU began piloting the Australian Spinal Cord Injury Register in one spinal unit early in 1995. During the pilot phase, the operation of the reporting system was tested: including data entry, quality assurance, and reporting functions. Patient unit record data, with personal identifiers, was transferred with patient agreement from the unit to NISU for newly incident cases of SCI and also readmitted prevalent cases. Upon completion of the pilot phase, the registration of incident cases became operational in all six units.

\section{Registration process}

Two groups of patients are admitted to spinal units: new incident cases (about 300 per year) and prevalent cases. From July 1, 1995 all new incident cases were registered. The registration process began with the completion of the first of two Registration forms. The scope of the first form included patient history, demographic information, clinical assessment of patients during their acute stage of SCI, and a description of the event that led to their SCI. A copy of this form was then sent to NISU to initiate the registration process. At discharge, the second Registration form was completed. This form recorded details of their clinical status at discharge and complications during the course of treatment. A copy was forwarded to NISU to complete the registration process.

It was recognised that the low incidence rate of SCI would restrict the utility of register in its early years unless retrospective data could be obtained. The existence of data from an earlier national collection suggested the possibility of extending retrospective collection to 1986. Following a thorough quality assurance check of the data collected from 1986 to 1991, that data was entered into the register, providing the records of nearly 4000 prevalent cases.

In order for the register to capture other prevalent cases, the registration status of each case was assessed as they were admitted to the spinal unit from July 1, 1995. If they were not already registered and not newly incident, they were registered. In this way, the coverage of the register could be progressively improved. For cases newly incident in the period 1992 to mid-1995, that were not already registered, a special case note review was conducted, and data collected and submitted. This meant that all cases newly incident in the period 1986 to 1995 presenting to spinal units were registered. This was completed in May 1999.

A readmission form was completed, for all readmitted cases that were already registered, in order to record current medical problems and outcomes. 
Quality assurance

Upon arrival at NISU, registration and readmission forms were checked for completeness and entered into a computerised data management system developed by NISU. The system was designed with numerous clerical and electronic data checks as well as automated coding and reporting facilities. In the event that a key data item was missing, such as admission date (essential for initiation of the computerised registration system), birth date or spinal diagnosis, annotated copies were returned to the source spinal unit for completion. Each quarter, unit specific reports (case level data lists and aggregated summaries) were provided to each spinal unit, along with a summary report of nationally aggregated data. The unit specific report highlighted the incomplete data fields for individual cases with a request for updating prior to completion of the next reporting period. Cases that were not updated remained on the 'incomplete list' of successive quarterly reports until rectified. The annual statistical report was not generated until the Directors had provided a sign-off that all cases were notified. These procedures were designed to ensure that reporting was as complete as possible. For cases admitted prior to 1 July 1995, not all data items were available from the case notes or from the previous data collection.

\section{Coverage of the register}

The register should identify each and every case of SCI due to trauma. The special case note review verified that all spinal unit cases from 1986 were registered. However, it was known that children suffering spinal cord damage, expected to be few in number, were generally managed in paediatric hospitals. ${ }^{17}$ Also it was suspected that other hospitals would manage cases with temporary deficits or dysfunctions that had resolved prior to transfer to a spinal unit.

There was no direct way to assess the coverage of the register. However, there was strong circumstantial evidence to support the view of the spinal unit Directors that very few adults with a persisting traumatic neurological deficit were not referred to their units, either immediately or after stabilisation at another hospital. If there were such cases, it was highly probable that some of them would eventually come to the attention of a spinal unit for the treatment of pressure sores, urinary tract infections or other complications. During the special case note review of more than 1000 cases admitted to a spinal unit no such cases were identified. This suggests that these cases, if any, were small in number.

The coverage of the register is therefore thought to be complete for persisting cases of SCI, to adults, from trauma. Cases that died prior to admission to a spinal unit have not been registered to date because until recently the ABS did not code disease diagnoses, such as SCI, from the death certificates. It is important to note that the size of the persisting case pool reflects the cumulative effects of the rate of incidence of SCI, the patient response to retrieval and treatment, and the rate of survival to discharge.

\section{Staffing}

As there is, on average, only one new case of SCI admitted to each spinal unit each week and about three readmissions, the register was initiated on the understanding that external funding would not be provided for data collection at the spinal units. The spinal unit Medical Registrars were allocated the responsibility for completion of the forms with the assistance of Resident Medical Officers (RMOs) and other staff. Frequent rotation of RMOs through the spinal units and the perceived low priority of paper work by these officers caused problems in a number of units. The most successful staffing combination is clerical support for routine administration combined with a Medical Registrar with research interests and expertise who can complete the clinical sections of the forms. Data collection is now a part of the routine duties of nominated staff in most units. Two of the units have continued to have ongoing difficulties in achieving the required demand for timely information.

The Registry, based at the Research Centre for Injury Studies, has two staff, each at the level of $10 \%$ of the full time equivalent: a Director responsible for the information and research program and an assistant responsible for spinal unit liaison and data entry.

\section{Operating costs}

The total cost to the Research Centre for Injury Studies of maintaining the register and providing the routine data reports, inclusive of salaries, expenses and overheads, is currently budgeted at about \$Aus20 000 per year, which equates to a cost of less than $\$ A$ us 100 per new incident case of traumatic SCI. However, the costs in earlier years, before the system was bedded down, were about double this figure. Added to the annual cost are the one-off costs of setting up the computerised data entry and data management system (estimated at \$Aus20 000); the time spent in liaison to develop the information system (estimated at \$Aus10 000); and the costs of special case note reviews in a number of States to register cases incident in the period 1991 to 1995 (estimated at \$Aus20 000). Over the 5 years that the register has been operating, a period in which all prevalent cases from 1986 have been registered, total expenses have been about \$Aus250 000, which is less than one tenth of $1 \%$ of the total community cost of the long term care of the newly incident cases of a single year. This has been a worthwhile investment.

\section{Discussion}

Australia is one of the few countries that have a national population-based register of SCI, and based on information available to the author, was the first to 
achieve this objective. The cost of establishing and maintaining the register is very small when the community cost of SCI is considered. Indeed these costs should be seen as an investment. The register affords the opportunity to fulfil important information and research objectives concerning, for example, assessment of the incidence, prevalence and trends in SCI, the causes and means of preventing SCI, and the health and welfare of the prevalent population, including an assessment of survival time and causes of death. This would not be possible however without the vision shown by leaders of the health bureaucracy, the Directors of the spinal units, and those responsible for the implementation of the development plan.

The data collected are sufficient for routine information needs concerning, for example, annual reporting of the incidence and trends in SCI and aetiological studies. However, for many research questions further information is needed and special studies are required to supplement the information available on the register.

The utility of the data is best demonstrated through its usage. An outline of the SCI information and research program of the Research Centre for Injury Studies (RCIS), and its National Injury Surveillance Unit, follows.

National incidence, prevalence and trends in SCI From 1997, the RCIS has published an annual statistical report on the national incidence of SCI. ${ }^{9,17,18}$ A detailed investigation of national trends in SCI from 1986 is underway and will be completed later in 2000 .

An assessment of the national prevalence of SCI is also planned. It will be based on a consideration of the annual incidence of SCI and survival. Estimation of incidence and survival prior to 1986 will be based on those Australian states for which complete registration data can be assured and will take into account the trends in the road injury and other leading causes of SCI.

\section{Causes of SCI}

Research into the causes of SCI is currently focused on the primary cause, road traffic crashes. There has been a dramatic decrease in road fatalities in Australia since the 1970's. ${ }^{19}$ Over the 10 years 1986-1995 road fatalities declined by $30 \%$. However, based on available data, SCI had not declined appreciably over this period. ${ }^{17}$

A review of the international research literature has been initiated to identify causal factors. In addition, crash information reported to police for the period 1988 to 1995 in South Australia has been linked with the register to study the relationships between crash factors and injury outcomes and to test some hypotheses about the causes of SCI in crashes. The study is similar to an earlier Australian study, conducted by Wigglesworth. ${ }^{20}$
Assessment of survival

Survival time and causes of death are being investigated. The register has been linked with the Australian National Deaths Index to determine which members of the SCI population had died, when they died and what caused their death. A literature review has also been initiated.

\section{Health and welfare of the prevalent population}

Although survival following SCI is increasing, suggesting that the prevalent population may be increasing, information about the health and welfare of this population is poor. ${ }^{21}$ In 1999 a study will be initiated to determine the most appropriate methodology for assessing and monitoring the health and welfare of the prevalent SCI population, the intention being to implement a data collection system in Australia based on the register, if possible.

\section{Prevention of SCI}

The register forms the basis for research studies into the prevention of SCI. A study has been undertaken into the effectiveness of helmets in preventing cervical SCI to motorcyclists. There has been only one other study of this problem reported in the literature. ${ }^{22,23}$

\section{Conclusions}

The main lessons to be learned from the Australian experience are: (1) that it is possible to design, implement and maintain a national population based register, at relatively small financial cost; and (2) that a register provides data for important information and research purposes. As it is unlikely that Australia has unique conditions for the development of a registry, this experience should encourage others. For such an objective to be met, there need to be specialist units for the treatment of all cases of SCI in a defined population and a high degree of enthusiasm of the Directors of these units to participate in data collection. Specific procedures, including case auditing, should be put in place to ensure that every case is registered. To avoid the potential to develop an unworkable system, by attempting to cater for all information needs, it is imperative that a Register focuses on a relatively small minimum data set. It should also have the capability to be extended at individual spinal units, and to be expanded through data linkage and supplementary data collection procedures. The case definition and minimum data set should conform to relevant international data standards in order to provide the opportunity for the international comparison of information leading to the diffusion of new ideas on the prevention and treatment of SCI throughout the world. 


\section{References}

1 Refshauge W (ed). Towards prevention of spinal cord injury. Menzies Foundation Technical Report, No. 1. Menzies Foundation: Melbourne, 1987.

2 Wigglesworth EC. Towards prevention of spinal cord injury: the role of a national register. Paraplegia 1988; 26: 389-392.

3 Blumer CE, Quine S. Surveillance of traumatic spinal cord injury in Australia: the identification of information needs. Spinal Cord 1996; 34: 639-643.

4 Refshauge W (ed). Towards a registry of spinal injury. Menzies Foundation Technical Report, No. 2. Menzies Foundation: Melbourne, 1988.

5 Walsh J. Costs of spinal cord injury in Australia. Paraplegia 1988; 26: $380-388$

6 Van Balen HG, Mulder T, Keyser A. Towards a disabilityoriented epidemiology of traumatic brain injury. Disabil Rehabil 1966; 18: $181-190$.

7 Samsa G, Patrick CH, Feussner JR. Long-term survival of veterans with traumatic spinal cord injury. Arch Neurol 1993; 50: 909-914.

8 Whiteneck GG et al. Mortality, morbidity, and psychosocial outcomes of persons spinal cord injured more than 20 years ago. Paraplegia 1992; 30: 617-630.

9 O'Connor PJ, Cripps RA. Spinal cord injury, Australia 1997/98. Australian Injury Prevention Bulletin 21, December 1998. Research Centre for Injury Studies, Flinders University of South Australia: Adelaide 1998.

10 Blumer CE. A review of the needs and opportunities for the surveillance of spinal cord injury. Australian Institute of Health and Welfare, National Injury Surveillance Unit: Adelaide 1995.

11 Thurman DJ et al. Guidelines for surveillance of central nervous system injury. US Department of Health and Human Services, Centers for Disease Control and Prevention: Atlanta 1995.
12 Maynard Jr FM et al. International standards for neurological and functional classification of spinal cord injury. American Spinal Injury Association. Spinal Cord 1997; 35: 266-274.

13 World Health Organization. International classification of diseases, 9th revision, clinical modification (ICD-9-CM). WHO: Geneva 1996.

14 Australian Institute of Health and Welfare. National Health Data Dictionary: version 6.0. Australian Institute of Health and Welfare: Canberra 1997.

15 Hamilton BB, Fuhrer MJ (eds). Rehabilitation outcomes: analysis and measurement. Brooks: Baltimore, 1987, pp 137-147.

16 Australian Institute of Health and Welfare. Annual report 1997. 98. AIHW: Canberra 1998, pp 79-98.

17 O'Connor PJ, Cripps RA. Spinal cord injury, Australia 1995/96. Australian Injury Prevention Bulletin 16, October 1997. Research Centre for Injury Studies, Flinders University of South Australia: Adelaide, 1997.

18 Cripps RA, O’Connor PJ. Spinal cord injury, Australia 1996/97. Australian Injury Prevention Bulletin 18, April 1998. Research Centre for Injury Studies, Flinders University of South Australia: Adelaide, 1998.

19 Federal Office of Road Safety. The history of road fatalities in Australia. Monograph 23. Australian Government Publishing Service: Canberra, 1988.

20 Wigglesworth EC. Motor vehicle crashes and spinal injury. Paraplegia 1992; 30: $543-549$.

21 McColl MA et al. Expectations of life and health among spinal cord injured adults. Spinal Cord 1997; 35: 818-828.

22 Yeo J. Five-year review of spinal cord injuries in motorcyclists. Med J Aust 1979; 2: 381.

23 Yeo J. Spinal cord injuries in motor cyclists. J Traffic Med 1979; 7: $37-39$. 